\title{
Do people with AIDS develop cancer at younger ages than the general population?
}

\author{
Meredith S Shiels*, Ruth M Pfeiffer, Eric A Engels \\ From $12^{\text {th }}$ International Conference on Malignancies in AIDS and Other Acquired Immunodeficiencies \\ (ICMAOI) \\ Bethesda, MD, USA. 26-27 April, 2010
}

\section{Background}

Previous studies have reported that age at cancer diagnosis is far younger among persons with HIV than in the general population, suggesting that cancer development is "accelerated" in persons with HIV. However, these estimates were influenced by differences in population age structures, since there are fewer older people with HIV at risk for developing cancer. We compared ages at cancer diagnosis among people with AIDS and the general population, after adjusting for underlying population age differences.

\section{Methods}

The HIV/AIDS Cancer Match study links 15 U.S. HIV/ AIDS and cancer registries. Using data from 338,349 persons with AIDS from 1980-2006, we compared observed cancers in persons with AIDS and the general population, and expected cancers calculated by applying general population rates from the cancer registry to followup time of persons with AIDS. Expected cancers represent cases that would occur in the general population if it had the same structure as the AIDS population, defined by age, sex, race, year, and registry. Median age at cancer diagnosis was estimated for each cancer.

\section{Results}

The proportion of person-time contributed by older persons (65+ years) was far smaller in the AIDS (1\%) than in the general population (13\%). Reflecting this difference, the median observed age at diagnosis for most cancers was $\sim 15-30$ years younger among people with AIDS than in the general population. However, after accounting for differences in age structure, observed and expected ages at diagnosis did not differ for most cancers. Observed ages at diagnosis were younger than expected $(\mathrm{p}<0.001)$ only for Kaposi sarcoma (median 37 vs. 44 years), non-Hodgkin lymphoma ( 39 years vs. 43 years), lung cancer ( 49 years vs. 53 years), and anal cancer ( 42 years vs. 45 years), and older for Hodgkin lymphoma (41 years vs. 38 years; $\mathrm{p}<0.001)$.

\section{Conclusions}

For most cancers, age at diagnosis is similar between people with AIDS and the general population, after accounting for the ages of the populations at risk. After controlling for differences in population structure, age at diagnosis remained slightly younger for only a few cancers among people with AIDS. These differences may reflect the effects of HIV in accelerating the development of some cancers where immunosuppression plays an important role. Alternatively, some age differences might reflect earlier exposure to other cancer risk factors among HIV-infected people (e.g., HPV infection, tobacco).

\section{Acknowledgements}

This article has been published as part of Infectious Agents and Cancer Volume 5 Supplement 1, 2010: Proceedings of the $12^{\text {th }}$ International Conference on Malignancies in AIDS and Other Acquired Immunodeficiencies (ICMAOI). The full contents of the supplement are available online at http://www.biomedcentral.com/1750-9378/5?issue=S1.

Published: 11 October 2010

doi:10.1186/1750-9378-5-S1-A22

Cite this article as: Shiels et al: Do people with AIDS develop cancer at younger ages than the general population?. Infectious Agents and Cancer 2010 5(Suppl 1):A22. 